For personal use only. Not to be reproduced without permission of the publisher (editorial@gabi-journal.net).

\section{Role of hospital clinical pharmacist in transplantation, and generic immunosuppressive therapies}

\author{
Andrea Devaney, BPharm, MRPharmS (IPresc), FFRPS, DipClinPharm, MSC
}

The pharmacist is an important patient advocate in the transplant multidisciplinary team and can support patients in their medication taking to improve medication adherence. Medicine optimization and patient information are also vital to this aim. For more than 20 years patients transplanted at our institution have undergone a structured post-operative self-medication training programme so that at discharge they are familiar with all their new transplant medications.

During 2010-2011 certain National Health Service drivers encouraged UK hospitals to seek and use best value immunosuppressive products in order to generate efficiency savings that could be reinvested back into clinical services to further improve patient care. This led our hospital to a) switch patients to generic mycophenolate mofetil and then b) commence a supervised controlled switch programme for established transplant recipients on Prograf-tacrolimus to be converted to Adoport-tacrolimus. In our experience, use of generic immunosuppressants in a controlled environment, to avoid inadvertent tacrolimus brand switching, is a safe process. Patient education and awareness is also paramount.

Keywords: Generics substitution, immunosuppressants, immunosuppressive, selfmedication, tacrolimus, transplantation

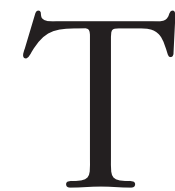
he most significant role of the hospital clinical pharmacist in patient care is risk minimization, and this can take many forms. Medicine optimization is a multidisciplinary and patient-focussed approach to getting the best use of medicines. Recent guidance has been introduced in the UK in an attempt to improve the quality and outcomes of medicines use. The four principles of this approach are to: aim to understand the patient's experience, ensure evidence-based choice of medicines, make medicines optimization part of routine practice and ensure medicines use is as safe as possible [1]. Through adoption of this medicines optimization and safety approach, adherence to treatment and reduction of medicine waste can be achieved by supporting patients to get the best outcome from their medicines. In addition, the pharmacist has a responsibility for ensuring cost-effective use of medicines and assisting in the management of the clinical units' drugs budget.
For over twenty years, all patients receiving a transplant at the Oxford Transplant Centre have undergone a period of medication training post-operatively during their routine inpatient stay. This remains current practice. Once the patient is stable postoperatively (usually post-operation day 2) they are given a patient specific medication card, see Figure 1. This card lists all their current medication, medication strengths, quantity to take throughout the day, reason for taking, and additional information including personalized stop dates of prophylactic medicines. This card is updated as changes are made on the ward and checked again for accuracy at the point of discharge. Patients can be sent their card template electronically, when requested. Additionally patients also receive a talk from the pharmacist who goes through the card and each medication listed explaining further information about the medication including side effects, how to take the medication and to alert patients to the medications they must avoid, now that they have received a transplant, e.g. NSAIDs (non-steroidal anti-inflammatory drugs), macrolide antibiotics. Patients are advised to always check with the transplant team before taking any new medicines prescribed by another healthcare professional. The pharmacist also explains how to obtain ongoing medication supplies. Once this talk is complete the patient can commence the supervised self-medication scheme. Here the nursing staff observes and supports the patient taking their own medications on the ward using their medication card. This provides opportunity for ongoing questions and education about their medication and also for the patient to be assessed actually taking their own medicines. After a minimum of two days of supervised medication taking if the nurse and patient are in agreement the patient can be assessed for 'solo' medication taking. The patient needs to complete a structured questionnaire about their medication, which is conducted by the nursing staff. If the patient is assessed as competent then they are permitted to go 'solo' and take their medication unsupervised, as they would do at home. All the patients' medication is locked in a patient's own drugs (POD) locker in the patient's room. When assessed to go 'solo' the patient also signs to take responsibility for their POD locker key. Each patient is also given a written discharge booklet during this time, which provides further information on their medication and practical information on going home safely with a new transplant.

This long standing multidisciplinary posttransplant self-medication scheme is valuable to patients and staff as it provides a unique opportunity to educate each patient about their new and vital medications at the time that they have received a transplant. In addition, it allows staff to assess patients taking their medication in real time and intervene appropriately where problems are identified, which may include medication regime simplification, or necessitate a multi-compartment compliance aid. In the case of the latter, the renal pharmacy team work closely with the patient/relative in an attempt to ensure all medication other than immunosuppressants are placed in the compliance aid. This permits flexibility of immunosuppressant dosing arising from clinic appointments, especially during the first few months post-transplant when

Author: Andrea Devaney, BPharm, MRPharmS (IPresc), FFRPS, DipClinPharm, MSc, Consultant Pharmacist Transplantation and Renal Services, Oxford University Hospitals NHS Trust, Oxford Transplant Centre, The Churchill, Headington, Oxford, OX3 7 LE, UK

Submitted: 12 November 2013; Revised: 18 December 2013; Accepted: 26 December 2013; Published online first: 10 January 2014 
Figure 1: Copy of medication record card used to initiate patient self-medication posttransplant

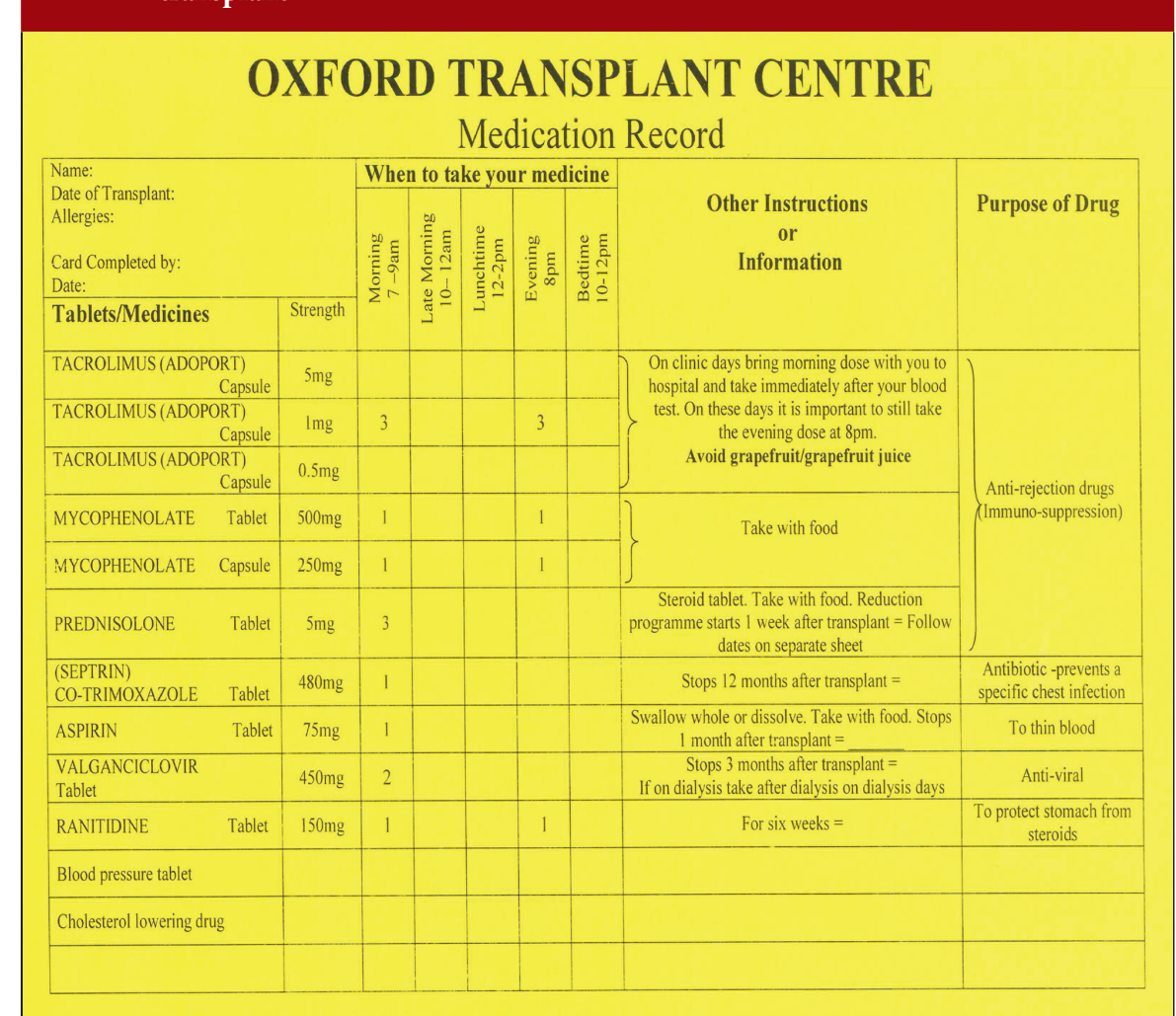

multiple dose changes are often required. The majority of the time this is achievable. The discharge nurse practitioners and outpatient transplant pharmacist are especially made aware of such instances so that there is a continuity of care from ward into the outpatient setting and that these patients receive any necessary ongoing support to ensure that they are managing and taking their medication effectively.

The Oxford Transplant Centre is a supraregional transplant centre and transplants patients from a large geographical area. Patients can receive a kidney, pancreas, islet, small bowel or modified multi-visceral transplant. Some of these transplant patients, due to geography, return to local care immediately on discharge with monthly follow-up in Oxford for a time. 900+ patients remain under long-term care at the Oxford Transplant Centre and as such continue to obtain all their transplant related mediation via transplant outpatient clinics at the hospital or via Oxford transplant satellite clinics at other hospitals in the surrounding area.

In 2001, we set up a medication home delivery service whereby as part of the patients' routine outpatient appointment to see their clinician (doctor or nurse practitioner) they also see the pharmacist in clinic, when they require medication. The pharmacist conducts a comprehensive medication review, impresses adherence, assesses stock levels of the medicines they have at home, ensures the computer medication records are accurate and up to date, checks the available biochemistry and haematology results, counsels the patient on any newly started medications, answers any medication related queries (from patient or member of multidisciplinary team) and, as a qualified independent non-medical prescriber writes the ongoing medication prescription that is required by the patient. Through impressing adherence the pharmacist has the opportunity to investigate any compliance issues with the patient and address these accordingly, e.g. changing a three times a day medication to an alternative once daily preparation if the patient is having difficulty taking all the daily doses. The prescriptions are then sent at the end of clinic, by the pharmacist, to an off-site homecare company who dispenses the prescription and sends the medication via the post (Royal Mail Special Delivery) to the patient's chosen address. Deliveries arrive within three working days of the clinic appointment. On average 400 prescriptions are issued each month to the homecare company from our transplant clinics. The outpatient transplant pharmacist is available in clinic five days a week. In addition to seeing patients the renal pharmacy team also offer a 'Medicines Triage Telephone' service. This service allows patients to access pharmacist advice easily for any medicine related queries. It is also used by other healthcare professionals (internal or external) requesting medicine advice for transplant recipients. We average 200 calls per month from this service. This service does not offer repeat prescriptions; patients must be seen in clinic in order to receive their ongoing transplant medication, unless there are exceptional, extenuating circumstances.

Generic immunosuppressive therapy is not a new concept and indeed has been in clinical practice for many years, e.g. prednisolone and azathioprine. In the UK in 2010 the government set out its vision for the future of the National Health Service (NHS) in the White Paper 'Equity and excellence: liberating the NHS' [2]. Within this paper Quality, Innovation, Productivity and Prevention (QIPP) work streams were described which would facilitate efficiency savings and these would then be reinvested back into clinical services to continually improve patient care; one of these work streams was medicine use and procurement. The opportunities to improve quality and effective use of funding by seeking best value in immunosuppressant spending was highlighted in a draft National Kidney Care QIPP plan (2011), which was then incorporated into the Kidney Care Commissioning Plan for 2012-2013 [3].

An NHS National tender for generic mycophenolate mofetil was awarded in February 2011. Mycophenolate mofetil (MMF) is not a critical dose drug and on the basis of this tender we switched all our patients on Cellcept to generic MMF via their next outpatient prescription, without event. The pharmacist advised and counselled each patient on this generic switch at the point of prescribing. No additional monitoring was implemented as a result of the switch. It is worth noting that at the 2013 American Transplant Congress an oral presentation was given by the Office of Generic Drugs, US Food and Drug Administration (FDA), on the pharmaceutical quality and bioequivalence of 11 different generic MMF tablets, in response to complaints received. This presentation concluded that all approved generic MMF products are well within FDA bioequivalence, there was 
no significant difference in manufacturing consistency despite different manufacturing processes and there was no suggestion efficacy and safety of generic MMF was compromised [4].

The first generic branded tacrolimus (Adoport, Sandoz) became available in the UK in June 2010. However, it was not until the national NHS tender for tacrolimus was awarded in summer 2011 that the uptake of generic tacrolimus started to increase.

At the Oxford Transplant Centre we started using generic tacrolimus (Adoport) de novo in August 2011, and then we commenced a supervised switch programme for established patients on Prograf-tacrolimus to be converted to Adoport-tacrolimus from October 2011. Posters were put in the clinic waiting areas alerting patients to this forthcoming change. We successfully switched over 400 patients during a 5-month period. We were fortunately placed to be already prescribing immunosuppressive therapy for these patients so implementation was not delayed. From the savings arising from this switch we seconded a nurse to help coordinate and manage the switch project for six months.

We designed a 'Dear Patient' letter, which was given to each patient ideally by the clinician seeing the patient that day at his or her outpatient appointment, or failing this, the pharmacist in clinic gave the patient the letter and explained the reason for the switch. The letter outlined what a generic medicine is, gave an everyday example of a widely used branded and generic medicine, e.g. Panadol-paracetamol, and explained that once patients had been switched they must remain on this brand. Patients were also reassured that although we were checking their blood levels 2-5 weeks after having commenced Adoport we were not expecting significant differences in blood tacrolimus levels, and this level was a precautionary measure. For some patients this meant they needed an extra bloods only visit to have a tacrolimus level measured, but for the majority of patients their check tacrolimus level was actually taken as part of their routine clinic visit. It was highlighted in the letter that any switch between brands must only be done under the supervision of the transplant unit. Patients are strongly encouraged to always check the brand that they receive in their medication home delivery and to contact us immediately if they have any concerns.
During the pharmacist consultation each patient was also given a Prograf-tacrolimus to Adoport-tacrolimus switch checklist. To prevent patients running out of their medication before their next outpatient appointment all patients are permitted and encouraged to keep a one-month supply of medication as reserve. This meant that at the time of issue of the first Adoport prescription most patients had a one-month stock of Prograf at home. It was stressed to the patient verbally and on the switch checklist sheet that they must only start Adoport once all the Prograf supply had been used. We recorded an estimated switch date on our database using the information provided to us by the patient about their stock supplies, during their outpatient appointment. The switch project nurse used this estimated date to follow up patients and ensure that they had had or were going to have a tacrolimus check level. It was imperative during the switch project that we knew when patients were having their tacrolimus check level taken so that it could be reviewed. Patients were also requested to either telephone or email us when they had had this check level taken. The switch project nurse was also the main contact for patients with any queries/concerns about the switch process. These queries were passed on as appropriate to the patient's clinician or the renal pharmacy team when further advice or action was necessary.

The real risk to patients from multiple brands of tacrolimus being widely available is inadvertent, uncontrolled substitution. It is our responsibility as healthcare professionals to alert patients to this and in turn to prescribe safely. Prescribing advice has been issued in the UK to ensure that tacrolimus is only prescribed and dispensed by brand name [5, 6]. In Europe, the Council of the European Society for Organ Transplantation published a report in 2011, which made similar recommendations [7]. This report also highlighted that generic immunosuppressive drugs which do not fulfil the stricter bioequivalence criteria should not be used. This specifically refers to generic ciclosporin products which were all granted a marketing authorization prior to the 2010 European Medicines Agency implementation of revised criteria for narrow therapeutic index drug bioequivalence $[8,9]$. The Medicines and Healthcare products Regulatory Agency (MHRA) have on file a Public Assessment
Report for each generic product, which contains the specific bioequivalence data for that product [10].

Our experience and that of many other transplant centres has shown that switching tacrolimus brands under a controlled environment, with appropriate measures in place to perform timely tacrolimus check blood levels, is first and foremost a safe process. Alloway et al. described a 2-week crossover study of Prograf-tacrolimus to Adoport-tacrolimus conversion or vice versa in stable renal transplant patients [11]. They concluded there was no statistical difference between the groups for Cmax, $\mathrm{C}_{0}$, Tmax or AUC at the end of each 14-day study period. Our experience mirrored this. Of 432 patients we switched over a 4-5-month period, 344 (80\%) had no dose change based on the 2-5-week 'check' level and there was no statistical difference in the mean tacrolimus level pre- and post-switch $(7.0 \mathrm{ng} / \mathrm{mL}$ and $7.47 \mathrm{ng} / \mathrm{mL}$ respectively). Fifty-two (12\%) patients had their tacrolimus dose changed at the time of switch so were excluded from the analysis. Thirty-six (8\%) patients had no coordinated 'check' level after their generics switch so were also excluded. We had no rejection episodes in our transplant centre attributable to the switch to generic immunosuppressant products. This generic therapy switch did however generate substantial efficiency savings and this has been reinvested back into clinical services to enhance patient care.

Hospital pharmacists are a vital part of the multidisciplinary healthcare team and are experts in medicines. The pharmacist, therefore, has a key role to play in supporting patients, as part of this team, to get the best outcome from their medicines. Through the patient-centred post-transplant self-medication scheme described and the follow-on of medicine provision via outpatient clinics, the pharmacist is able to build relationships with the patient and in so doing explore adherence, medicine understanding and optimize medicine use.

\section{Acknowledgement}

The author would like to thank the Oxford Transplant Centre renal pharmacy team and Ms Nicola Keats (switch project nurse) for their assistance in collecting the generic switch data and especially Mr Paul Clarke for his help with the analysis. 
Competing interests: During the last 12 months, the author received sponsorship from Sandoz to attend an international transplant conference (registration, flight and accommodation). In addition, the author has received a speaker's honorarium from Sandoz for an oral presentation on the Oxford experience of generic immunosuppressant.

Provenance and peer review: Commissioned; externally peer reviewed.

\section{References}

1. Royal Pharmaceutical Society. Medicines optimisation: helping patients to make the most of medicines. May 2013 [homepage on the Internet]. $2010 \mathrm{Apr}$ [cited 2013 Dec 18]. Available from: http://www. rpharms.com/promoting-pharmacy-pdfs/helpingpatients-make-the-most-of-their-medicines.pdf

2. Department of Health. Equity and excellence: liberating the NHS. July 2010 [homepage on the Internet]. 2010 Jul [cited 2013 Dec 18]. Available from: https://www.gov.uk/government/ publications/liberating-the-nhs-white-paper
3. NHS Specialised Commissioning Transition. Kidney Care Commissioning - opportunities for driving improvements in quality and productivity in kidney care services. A transitional plan for 2012-2013. April 2012 [homepage on the Internet]. 2012 May [cited 2013 Dec 18]. Available from: www.kidney. org.uk/reference

4. Jiang W, Lionberger R, Li J, et al. Pharmaceutical quality and bioequivalence assessment of generic mycophenolate mofetil tablets (Abstract:\#418). Am J Transplant. 2013;13 Suppl 5:161.

5. Joint Formulary Committee. British National Formulary. 65th ed. London: BMJ Group and Pharmaceutical Press; 2013.

6. Medicines and Healthcare products Regulatory Agency (MHRA). Oral tacrolimus products: prescribe and dispense by brand name only, to minimise the risk of inadvertent switching between products, which has been associated with reports of toxicity and graft rejection. Drug safety update. June 2012;5(11):A1.

7. van Gelder T. ESOT Advisory Committee on Generic Substitution. European Society for Organ Transplantation Advisory Committee recommendations on generic substitution of immunosuppressive drugs. Transpl Int. 2011;24(12):1135-41.
8. European Medicines Agency. Committee for Medicinal Products for Human Use (CHMP). Guideline on the investigation of bioequivalence. 20 January 2010 [homepage on the Internet]. 2010 [cited 2013 Dec 18]. Available from: http://www. ema.europa.eu/docs/en_GB/document_library/ Scientific_guideline/2010/01/WC500070039.pdf

9. Maliepaard M, Leufkens HGM, Yang Yu. Equivalence of generic medicines in general and immunosuppressants in particular - a regulatory opinion on switching of cyclosporin, tacrolimus and mycophenolate mofetil. Generics and Biosimilars Initiative Journal (GaBI Journal). 2013; 2(2):86-90. doi:10.5639/gabij.2013.0202.019

10. Medicines and Healthcare products Regulatory Agency (MHRA). Public Assessment Reports for medicines: A-Z listing [homepage on the Internet]. 2013 [cited 2013 Dec 18]. Available from: http:// www.mhra.gov.uk/Publications/PublicAssessmentReports/A-Zlisting/index.htm?indexChar=C

11. Alloway RR, Sadaka B, Trofe-Clark J, et al. A randomised pharmacokinetic study of generic tacrolimus versus reference tacrolimus in kidney transplant recipients. Am J Transplant. 2012;12(10):2825-31.

DOI: 10.5639/gabij.2014.0302.023

Copyright @ 2014 Pro Pharma Communications International 